\title{
PROPER ISOPARAMETRIC SEMI-RIEMANNIAN SUBMANIFOLDS IN A SEMI-RIEMANNIAN SPACE FORM
}

By

\author{
Naoyuki KoIKE
}

\section{§ 0. Introduction.}

In a sphere, Erbacher [2] and Yano-Ishihara [14] characterized Riemannian submanifolds with non-negative sectional curvature, flat normal connection and parallel mean curvature vector under the additional assumptions. It is a natural question to consider this problem in the semi-Riemannian case. Recently, we characterized proper isoparametric semi-Riemannian hypersurfaces in a semiRiemannian space form under certain assumptions [1]. The main purpose of this paper is to characterize, in a semi-Riemannian space form, proper isoparametric semi-Riemannian submanifolds with non-negative (or non-positive) sectional curvature and parallel mean curvature vector under certain additional assumptions.

The author wishes to express his gratitude to Professor S. Yamaguchi for his constant encouragement and various advice. He also wishes to thank Professor N. Abe for his helpful suggestions.

\section{§1. Preliminaries.}

Throughout this paper, all manifolds are smooth and connected and geometrical objects are assumed to be smooth unless mentioned otherwise. In this section, we prepare basic facts about semi-Riemannian submanifolds in a semiRiemannian. manifold. We call a non-degenerate symmetric $(0,2)$-tensor field on an $n$-dimensional manifold $M^{n}$ a semi-Riemannian metric of $M^{n}$ and a manifold $M^{n}$ equipped with such a metric a semi-Riemannian manifold. Especially, an $n$-dimensional real vector space equipped with a non-degenerate symmetric bilinear form of signature $(\nu, n-\nu)$ given by

$$
\langle x, x\rangle=-\sum_{i=1}^{\nu} x_{i}{ }^{2}+\sum_{j=\nu+1}^{n} x_{j}^{2}
$$

Received May 2, 1988. 
is called an $n$-dimensional semi-Euclidean space and is denoted by $R_{\nu}^{n}$, where $x=\left(x_{1}, \cdots, x_{n}\right)$ is the natural coordinate. A frame $\left(e_{1}, \cdots, e_{n}\right)$ is said to be orthonormal if $\left|\left\langle e_{i}, e_{j}\right\rangle\right|=\delta_{i j}$. Semi-Riemannian manifolds $S_{\nu}^{n}(c)$ and $H_{\nu}^{n}(c)$ given by

$$
\begin{array}{ll}
S_{\nu}^{n}(c)=\left\{\left(x_{1}, \cdots, x_{n+1}\right) \in R_{\nu}^{n+1} \mid-\sum_{i=1}^{\nu} x_{i}{ }^{2}+\sum_{i=\nu+1}^{n+1} x_{i}{ }^{2}=1 / c\right\} & (c>0), \\
H_{\nu}^{n}(c)=\left\{\left(x_{1}, \cdots, x_{n+1}\right) \in R_{\nu+1}^{n+1} \mid-\sum_{i=1}^{\nu+1} x_{i}{ }^{2}+\sum_{i=\nu+2}^{n+1} x_{i}{ }^{2}=1 / c\right\} \quad(c<0)
\end{array}
$$

are called a semi-sphere and a semi-hyperbolic space, respectively. These spaces are complete and of constant curvature $c$, that is,

$$
R(X, Y) Z=c(X \wedge Y) Z(=c(\langle Y, Z\rangle X-\langle X, Z\rangle Y)),
$$

where $R$ is the curvature tensor $(n \geqq 2)$. It is clear that $S_{\nu}^{n}(c)$ is diffeomorphic to $R^{\nu} \times S^{n-\nu}$ and $H_{\nu}^{n}(c)$ is diffeomorphic to $S^{\nu} \times R^{n-\nu}$, where $S^{\mu}=S_{0}^{\mu}$ and $R^{\mu}=R_{0}^{\mu}$. We note that $S_{n}^{n}(c)$ and $H_{0}^{n}(c)$ are not connected and $S_{n-1}^{n}(c)$ and $H_{1}^{n}(c)$ are not simply connected. We call these three spaces $R_{\nu}^{n}, S_{\nu}^{n}(c)$ and $H_{\nu}^{n}(c)$ semi-Riemannian space forms.

A semi-Riemannian manifold $M^{n}$ isometrically immersed into a semi-Riemannian manifold $\tilde{M}^{m}$ by an immersion $f$ is called a semi-Riemannian submanifold of $\tilde{M}$. Since $f$ can be treated locally as an imbedding, $p(\in M)$ will often be identified with $f(p)$ and the mention of $f$ will be supressed. Especially if $n=m-1$, then $M$ is called a semi-Riemannian hypersurface of $\tilde{M}$. Let $T_{p} M$ (resp. $T_{p}^{1} M$ ) be the tangent space (resp. the normal space) of $M$ at $p \in M, T M$ (resp. $T^{\perp} M$ ) the tangent bundle (resp. the normal bundle) of $M$ and $\Gamma(T M)$ resp. $\Gamma\left(T^{\perp} M\right)$ ) the space of all cross sections of $T M$ (resp. $T^{\perp} M$ ). We denote the semi-Riemannian metrics of $\tilde{M}$ and $M$ by $\langle$,$\rangle and the Levi-Civita connec-$ tions on $\tilde{M}$ (resp. $M$ ) by $\tilde{\nabla}$ (resp. $\nabla$ ). For any $X \in T M$ and any $Y \in \Gamma(T M)$, we have the Gauss formula:

$$
\tilde{\nabla}_{X} Y=\nabla_{X} Y+h(X, Y),
$$

where $\nabla_{X} Y$ and $h(X, Y)$ are the tangential and the normal components of $\tilde{\nabla}_{X} Y$ respectively. It is easy to show that $h$ is symmetric. We call $h$ the second fundamental form of the semi-Riemannian submanifold $M$.

For any $X \in T M$ and any $E \in \Gamma\left(T^{\perp} M\right)$, we have the Weingarten formula:

$$
\tilde{\nabla}_{X} E=-A_{E} X+\nabla_{X}^{1} E,
$$

where $-A_{E} X$ and $\nabla_{X}^{\perp} E$ are the tangential and the normal components of $\tilde{\nabla}_{X} E$ respectively. It is easy to verify that $\nabla^{\perp}$ is a connection of the normal bundle of $M$. We call $A$ the shape operator of the semi-Riemannian submanifold $M$. 
It follows that

$$
\langle h(X, Y), E\rangle=\left\langle A_{E} X, Y\right\rangle
$$

for any $X, Y \in T_{p} M$ and any $E \in T_{p}^{\perp} M(p \in M)$.

Let $\tilde{R}$ and $R$ be the curvature tensors of $\tilde{M}$ and $M$, respectively. The equation of Gauss is given by

$$
R(X, Y) Z=(\tilde{R}(X, Y) Z)^{T}+\sum_{a=1}^{m-n} \varepsilon_{a}^{1}\left(A_{E_{a}} X \wedge A_{E_{a}} Y\right) Z \quad\left(\varepsilon_{a}^{1}=\left\langle E_{a}, E_{a}\right\rangle\right)
$$

for any $X, Y$ and $Z \in T_{p} M(p \in M)$, where $(\tilde{R}(X, Y) Z)^{T}$ is the tangential component and $\left(E_{1}, \cdots, E_{m-n}\right)$ is an orthonormal frame of $T_{p}^{+} M$. The equation of Codazzi is given by

$$
(\tilde{R}(X, Y) E)^{T}=\left(\nabla_{Y}^{\prime} A\right)_{E} X-\left(\nabla_{X}^{\prime} A\right)_{E} Y
$$

for any $X, Y \in T_{p} M$ and any $E \in T_{p}^{\perp} M(p \in M)$, where $\left(\nabla_{X}^{\prime} A\right)_{E} Y=\nabla_{X}\left(A_{E} Y\right)$ $A_{\nabla_{X}^{1} E} Y-A_{E}\left(\nabla_{X} Y\right)$. In particular, if $\tilde{M}$ is of constant curvature $\tilde{c}$, then these equations can be rewritten as follows:

$$
\begin{gathered}
R(X, Y)=\tilde{c} X \wedge Y+\sum_{a=1}^{m-n} \varepsilon_{a}^{1} A_{E_{a}} X \wedge A_{E_{a}} Y \\
\left(\nabla_{X}^{\prime} A\right)_{E} Y=\left(\nabla_{Y}^{\prime} A\right)_{E} X .
\end{gathered}
$$

\section{$\S 2$. Shape operators of proper isoparametric semi-Riemannian submanifolds.}

Let $Q$ be a $(1,1)$-tensor of a real vector space $V$ equipped with a nondegenerate symmetric bilinear form. If $Q$ can be expressed by a real diagonal matrix with respect to an orthonormal frame of $V$, then $Q$ is said to be proper.

LeMMA 2.1. Let $Q_{1}, \cdots, Q_{k}$ be proper $(1,1)$-tensors of $V$ such that $\left[Q_{a}, Q_{b}\right]$ $=0(1 \leqq a, b \leqq k)$. Then $Q_{1}, \cdots, Q_{k}$ are simultaneously diagonalizable with respect to an orthonormal frame of $V$.

PROOF. It is sufficient to show the case where $k=2$. Let $\left\{\lambda_{1}, \cdots, \lambda_{t}\right\}$ (resp. $\left.\left\{\mu_{1}, \cdots, \mu_{u}\right\}\right)$ be the set of all distinct eigenvalues of $Q_{1}$ (resp. $Q_{2}$ ). Set $V_{\lambda_{a}}=$ $\operatorname{Ker}\left(Q_{1}-\lambda_{a} I\right)(1 \leqq a \leqq t), W_{\mu_{b}}=\operatorname{Ker}\left(Q_{2}-\mu_{b} I\right)(1 \leqq b \leqq u)$. Let $v$ be a vector of $V_{\lambda_{a}}$. There exists a unique $v_{b} \in W_{\mu_{b}}(1 \leqq b \leqq u)$ such that $v=v_{1}+\cdots+v_{u}$ because of $V=\bigoplus_{1 \leq b \leq u} W_{\mu_{b}}$, where $\oplus$ means the orthogonal direct sum. By operating $Q_{1}$ to both sides of $v=v_{1}+\cdots+v_{u}$, we have $\lambda_{a} v_{1}+\cdots+\lambda_{a} v_{u}=Q_{1} v_{1}+\cdots+Q_{1} v_{u}$. On the other hand, from $\left[Q_{1}, Q_{2}\right]=0$, it follows that $Q_{1} v_{b} \in W_{\mu_{b}}(1 \leqq b \leqq u)$. Hence, we have $Q_{1} v_{b}=\lambda_{a} v_{b}$, which means that $v_{b} \in V_{\lambda_{a}} \cap W_{\mu_{b}}$. Therefore, we can obtain 
$V_{\lambda_{a}}=\bigoplus_{b \in G_{a}}\left(V_{\lambda_{a}} \cap W_{\mu_{b}}\right)$ and hence $V=\bigoplus_{(a, b) \in G}\left(V_{\lambda_{a}} \cap W_{\mu_{b}}\right)$ because of $V=\underset{1 \leq a \leq t}{\bigoplus_{a}} V_{\lambda_{a}}$, where $G=\left\{(a, b) \mid 1 \leqq a \leqq t, 1 \leqq b \leqq u,\left(V_{\lambda_{a}} \cap W_{\mu_{b}} \neq\{0\}\right)\right\} \quad$ and $G_{a}=\{b \mid(a, b) \in G\}$ $(1 \leqq a \leqq t)$. Moreover, since $V_{\lambda_{a}} \cap W_{\mu_{b}}((a, b) \in G)$ are orthogonal to one another, they are non-degenerate, respectively. So we can take orthonormal frames of $V_{\lambda_{a}} \cap W_{\mu_{b}}((a, b) \in G)$ and, by using them, we can construct an orthonormal frame of $V$. It is clear that $Q_{1}$ and $Q_{2}$ are simultaneously diagonalizable with respect to this orthonormal frame. This completes the proof. Q.E.D.

Let $A$ be the shape operator of a semi-Riemannian submanifold $M$ of a semi-Riemannian manifold $\tilde{M}$. The submanifold $M$ is said to be proper if $A_{E}$ is proper for any $E \in T^{\perp} M$. If the normal connection is flat and the characteristic polynomial of $A_{E}$ is constant over the domain of $E$ for any local parallel normal vector field $E$, then $M$ is said to be $i$ soparametric $[3,11]$. By a similar method to the proof of Lemma 2 in [2], we can show the following.

LEMMA 2.2. Let $M^{n}$ be a proper semi-Riemannian submanifold in a semiRiemannian space form $\tilde{M}^{n+r}$ of constant curvature $\tilde{c}$ with flat normal connection and parallel mean curvature vector. Then we have

$$
\Delta\langle A, A\rangle=2\left\langle\nabla^{\prime} A, \nabla^{\prime} A\right\rangle+\sum_{i, j=1}^{n} \sum_{a=1}^{r} K_{i j}\left(\lambda_{i}^{a}-\lambda_{j}^{a}\right)^{2}\left\langle E_{a}, E_{a}\right\rangle,
$$

where $\left(e_{1}, \cdots, e_{n}\right)$ and $\left(E_{1}, \cdots, E_{r}\right)$ are an orthonormal tangent frame and an

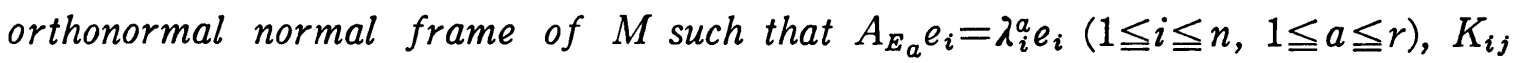
is the sectional curvature with respect to the 2-dimensional subspace spanned by $e_{i}$ and $e_{j}(i \neq j)$, and $\Delta$ is the Laplacian operator of $M$.

Note that $\langle A, A\rangle$ and $\left\langle\nabla^{\prime} A, \nabla^{\prime} A\right\rangle$ are defined as follows:

$$
\begin{aligned}
& \langle A, A\rangle=\sum_{i=1}^{n} \sum_{a=1}^{r} \varepsilon_{i} \varepsilon_{a}^{1}\left\langle A_{E_{a}} e_{i}, A_{E_{a}} e_{i}\right\rangle \quad \text { and } \\
& \left\langle\nabla^{\prime} A, \nabla^{\prime} A\right\rangle=\sum_{i, j=1}^{n} \sum_{a=1}^{r} \varepsilon_{i} \varepsilon_{j} \varepsilon_{a}^{1}\left\langle\left(\nabla_{e_{i}}^{\prime} A\right)_{E_{a}} e_{j},\left(\nabla_{e_{i}}^{\prime} A\right)_{E_{a}} e_{j}\right\rangle,
\end{aligned}
$$

where $\varepsilon_{i}=\left\langle e_{i}, e_{i}\right\rangle(1 \leqq i \leqq n)$ and $\varepsilon_{a}^{1}=\left\langle E_{a}, E_{a}\right\rangle(1 \leqq a \leqq r)$.

We denote by $B_{1} \oplus \cdots \oplus B_{\imath}$ the following matrix:

$$
\left(\begin{array}{cccc}
B_{1} & & & \\
& \ddots & 0 \\
& 0 & \ddots & \\
& & & B_{l}
\end{array}\right)
$$

where $B_{i}(1 \leqq i \leqq l)$ are square matrices, respectively. 
By using Lemma 2.1 and 2.2, we can show the following theorem.

THEOREM 2.3. Let $M^{n}$ be a proper isoparametric semi-Riemannian submanifold in $R_{\nu}^{n+r}$ with parallel mean curvature vector and $\left\langle\nabla^{\prime} A, \nabla^{\prime} A\right\rangle \geqq 0$. Furthermore, suppose that all sectional curvatures of $M$ are non-negative (resp. nonpositive) and $\left.\langle\rangle\right|_{,T^{\perp_{M}}}$ is positive definite (resp. negative definite). Then, for any point $p$ of $M$, there exists a parallel orthonormal normal frame field $\left(E_{1}, \cdots, E_{r}\right)$ on a neighborhood $U$ of $p$ with the property (\#): At each point of $U, A_{E_{1}}, \cdots, A_{E_{r}}$ can be expressed with respect to a certain orthonormal tangent frame $\left(e_{1}, \cdots, e_{n}\right)$ as follows:

$$
\begin{aligned}
& A_{E_{1}}=\lambda_{1} I_{l_{1}} \oplus 0_{k_{1}}, \\
& A_{E_{2}}=0_{l_{1}} \oplus \lambda_{2} I_{l_{2}} \oplus 0_{k_{2}}, \\
& \cdots \cdots, \\
& A_{E_{s}}=\left(\bigoplus_{a=1}^{s-1} 0_{l_{a}}\right) \oplus \lambda_{s} I_{l_{s}} \oplus 0_{k_{s}}, \\
& A_{E_{s+1}}=\cdots=A_{E_{r}}=0,
\end{aligned}
$$

where $\lambda_{a} \neq 0, k_{a}=n-\sum_{b=1}^{a} l_{b}, l_{a} \geqq 1(1 \leqq a \leqq s), k_{s} \geqq 0$ and $0_{l}$ and $I_{l}$ are the zero matrix of type $(l, l)$ and the identity matrix of type $(l, l)$, respectively.

Proof. Fix a point $p$ of $M$. Since the normal connection of $M$ is flat, there exists a parallel orthonormal normal frame field $\left(E_{1}, \cdots, E_{r}\right)$ on a neighborhood $U$ of $p$ and moreover $\left[A_{E_{a}}, A_{E_{b}}\right]=0$ holds $(1 \leqq a, b \leqq r)$. Hence, by Lemma 2. $1, A_{E_{1}}, \cdots, A_{E_{r}}$ are simultaneously diagonalizable with respect to an orthonormal tangent frame at each point of $U$. Suppose that $A_{E_{1}}, \cdots, A_{E_{r}}$ are expressed with respect to an orthonormal tangent frame $\left(e_{1}, \cdots, e_{n}\right)$ at each point of $U$ as follows:

$$
A_{E_{1}}=\lambda_{1}^{1} I_{1} \oplus \cdots \oplus \lambda_{n}^{1} I_{1}, \cdots, A_{E_{r}}=\lambda_{1}^{r} I_{1} \oplus \cdots \oplus \lambda_{n}^{r} I_{1} .
$$

By our assumptions and Lemma 2.2, we have

$$
K_{i j}\left(\lambda_{i}^{a}-\lambda_{j}^{a}\right)^{2}=0(1 \leqq a \leqq r, 1 \leqq i \neq j \leqq n) .
$$

In the first place, suppose that $p$ is a geodesic point, that is, $A_{E_{1}}=\cdots=A_{E_{r}}$ $=0$ at $p$. Since $M$ is isoparametric, $A_{E_{1}}=\cdots=A_{E_{r}}=0$ on $U$. Thus $\left(E_{1}, \cdots, E_{r}\right)$ satisfies the property (\#).

In the next place, we consider the case where $p$ is not a geodesic point. Since $p$ is not a geodesic point, we may assume that $\lambda_{1}^{1} \neq 0, K_{1 i} \neq 0\left(2 \leqq i \leqq l_{1}\right)$ and $K_{1 j}=0\left(l_{1}+1 \leqq j \leqq n\right)$. From (2.1), we have 


$$
\lambda_{1}^{a}=\lambda_{i}^{a}\left(2 \leqq i \leqq l_{1}, 1 \leqq a \leqq r\right)
$$

We set

$$
\begin{gathered}
E_{1}^{\prime}:=\left(\sum_{a=1}^{r} \lambda_{1}^{a} E_{a}\right) / \lambda_{1}, \\
\bar{E}_{b}:=\left(\lambda_{1}^{1} E_{b}-\lambda_{1}^{b} E_{1}\right) /\left(\left(\lambda_{1}^{1}\right)^{2}+\left(\lambda_{1}^{b}\right)^{2}\right)^{1 / 2} \quad(2 \leqq b \leqq r),
\end{gathered}
$$

where $\lambda_{1}=\left(\sum_{a=1}^{r}\left(\lambda_{1}^{a}\right)^{2}\right)^{1 / 2}$. It is clear that

$$
\left\langle E_{1}^{\prime}, E_{1}^{\prime}\right\rangle= \pm 1,\left\langle E_{1}^{\prime}, \bar{E}_{b}\right\rangle=0,\left\langle\bar{E}_{b}, \bar{E}_{b}\right\rangle= \pm 1, \quad \nabla^{\perp} E_{1}^{\prime}=\nabla^{\perp} \bar{E}_{b}=0 .
$$

Because of (2.2), $A_{E_{1}^{\prime}}$ and $A_{\bar{E}_{b}}(2 \leqq b \leqq r)$ are expressed as follows :

$$
\begin{aligned}
& A_{E_{1}^{\prime}}=\lambda_{1} I_{l_{1}} \oplus \lambda^{\prime}{ }_{l_{1}+1} I_{1} \oplus \cdots \oplus \lambda^{\prime \prime}{ }_{n} I_{1} \\
& A_{\bar{E}_{b}}=0_{l_{1}} \oplus \bar{\lambda}_{l_{1}+1}^{b} I_{1} \oplus \cdots \oplus \bar{\lambda}_{n}^{b} I_{1} \quad(2 \leqq b \leqq r) .
\end{aligned}
$$

Let $\left(E_{2}^{\prime}, \cdots, E_{r}^{\prime}\right)$ be an orthonormal normal system given by applying GramSchmidt orthogonalization to $\left(\bar{E}_{2}, \cdots, \bar{E}_{r}\right)$. It is clear that $E_{b}^{\prime}(2 \leqq b \leqq r)$ are parallel and $A_{E_{b}^{\prime}}(2 \leqq b \leqq r)$ are expressed as follows:

$$
A_{E_{b}^{\prime}}=0_{l_{1}} \oplus \lambda_{l_{1}+1}^{b} I_{1} \oplus \cdots \oplus \lambda_{n}^{\prime b} b_{1} \quad(2 \leqq b \leqq r) .
$$

By the assumption that $K_{1 i}=0\left(l_{1}+1 \leqq i \leqq n\right)$ and the equation (1.4), we have

$$
\begin{aligned}
0=K_{1 i} & =\left\langle e_{1}, e_{1}\right\rangle\left\langle e_{i}, e_{i}\right\rangle\left\langle R\left(e_{1}, e_{i}\right) e_{i}, e_{1}\right\rangle \\
& =\left\langle e_{1}, e_{1}\right\rangle\left\langle e_{i}, e_{i}\right\rangle\left\langle \pm \sum_{a=1}^{r}\left(A_{E_{a}^{\prime}} e_{1} \wedge A_{E_{a}^{\prime}} e_{i}\right) e_{i}, e_{1}\right\rangle \\
& = \pm \lambda_{1} \lambda^{\prime 1}{ }_{i},
\end{aligned}
$$

that is, $\lambda^{\prime 1}=0\left(l_{1}+1 \leqq i \leqq n\right)$. After all, we obtain $A_{E_{1}^{\prime}}=\lambda_{1} I_{l_{1}} \oplus 0_{n-l_{1}}$. Thus if $A_{E_{2}^{\prime}}=\cdots=A_{E_{r}^{\prime}}=0,\left(E_{1}^{\prime}, \cdots, E_{r}^{\prime}\right)$ satisfy the property (\#). So we consider the case where there exists $b \geqq 2$ such that $A_{E_{b}^{\prime}} \neq 0$. We may assume that $\lambda_{l_{1}+1}^{2}$ $\neq 0, K_{l_{1}+1, i} \neq 0\left(l_{1}+2 \leqq i \leqq l_{1}+l_{2}\right)$ and $K_{l_{1}+1, j}=0\left(l_{1}+l_{2}+1 \leqq j \leqq n\right)$. By the same process as the above, we can obtain a parallel orthonormal normal system $\left(E_{2}^{\prime \prime}, \cdots, E_{r}^{\prime \prime}\right)$ such that

$$
\begin{aligned}
& A_{E_{2}^{\prime \prime}}=0_{l_{1}} \oplus \lambda_{2} I_{l_{2}} \oplus 0_{n-l_{1}-l_{2}}, \\
& A_{E_{b}^{\prime \prime}}=0_{l_{1}+l_{2}} \oplus \lambda_{l_{1}+l_{2}+1}^{\prime b} I_{1} \oplus \cdots \oplus \lambda^{\prime \prime}{ }_{n}^{b} I_{1} \quad(3 \leqq b \leqq r) .
\end{aligned}
$$

In the sequel, by repeating the same process, we reach the conclusion. Q.E.D.

In general, if $M$ is simply connected and the normal connection is flat, then there exists a parallel orthonormal normal frame field on $M$. By using this fact, we can obtain the following. 
THEOREM 2.4. Under the same hypothesis as in Theorem 2.3, if $M$ is simply connected, then there exists a parallel orthonormal normal frame field $\left(E_{1}, \cdots, E_{r}\right)$ on $M$ with the property (\#) in Theorem 2.3.

\section{§3. Eigendistributions of the shape operator.}

Let $M$ be a semi-Riemannian manifold equipped with a metric $\langle$,$\rangle and D$ a distribution on $M$, that is, a subbundle of the tangent bundle $T M$. If $\nabla_{X} Y \in D$ for any $X \in T M$ and any $Y \in \Gamma(D)$, then $D$ is said to be parallel, where $\Gamma(D)$ is the space of all cross sections of $D$. If $\left.\langle\rangle\right|_{D$,$} is non-degenerate at each$ point of $M$, then $D$ is said to be non-degenerate. We have

LEMMA A. Let $D$ be a non-degenerate parallel distribution on a semi-Riemannian manifold $M$. Let $M^{\prime}$ be the maximal integral manifold of $D$ through a point of $M$. Then $M^{\prime}$ is a totally geodesic semi-Riemannian submanifold of $M$. If $M$ is complete, then so is $M^{\prime}$.

Let $Q$ be a $(1,1)$-tensor field on $M$. If $Q$ is proper at each point of $M$, then $Q$ is said to be proper. The following result is stated in [1].

LEMMA B. Let $Q$ be a proper $(1,1)$-tensor field on $M$ which has exactly two mutually distinct constant eigenvalues $\lambda_{1}$ and $\lambda_{2}$. Suppose that $\left(\nabla_{X} Q\right) Y=\left(\nabla_{Y} Q\right) X$ holds for any $X, Y \in T_{p} M(p \in M)$. Then $D_{\lambda_{i}}=\operatorname{Ker}\left(Q-\lambda_{i} I\right)(i=1,2)$ are nondegenerate parallel distributions on $M$.

By using these results, we obtain the following theorem.

THEOREM 3.1. Let $M^{n}$ be a semi-Riemannian submanifold of $R_{\nu}^{n+r}$. Suppose that for each point $p$ of $M$, there exists a parallel orthonormal normal frame field $\left(E_{1}, \cdots, E_{r}\right)$ on a neighborhood $U$ of $p$ with the property (\#) in Theorem 2.3. Then

(i) $D_{a}=\operatorname{Ker}\left(A_{E_{a}}-\lambda_{a} I\right)(1 \leqq a \leqq s)$ and $D_{0}=\left(D_{1} \oplus \cdots \oplus D_{s}\right)^{\perp}$ are parallel on $U$ respectively, where $\left(D_{1} \oplus \cdots \oplus D_{s}\right)^{\perp}$ is the orthogonal complement of $D_{1} \oplus \cdots \oplus D_{s}$ in $T U$,

(ii) the each maximal integral manifold of $D_{a}$ is a totally umbilical submanifold of $R_{\nu}^{n+r}$ with the mean curvature vector $\varepsilon_{a}^{\perp} \lambda_{a} E_{a}\left(\varepsilon_{a}^{1}=\left\langle E_{a}, E_{a}\right\rangle\right)(1 \leqq a \leqq s)$ and that of $D_{0}$ is a totally geodesic semi-Riemannian submanifold of $R_{\nu}^{n+r}$.

Proof. Let us restrict ourselves to the neighborhood $U$.

(i) By applying Lemma B to $A_{E_{a}}$, we see that each $D_{a}$ is parallel on $U$ 
$(1 \leqq a \leqq s)$. Since $D_{1} \oplus \cdots \oplus D_{s}$ is parallel on $U$, so is the orthogonal complement $D_{0}$.

(ii) Let $U_{(a)}$ be the maximal integral manifold of $D_{a}$ through a point of $U(1 \leqq a \leqq s)$. We denote the second fundamental form of $U$ (resp. $U_{(a)}$ ) in $R_{\nu}^{n+r}$ by $h$ (resp. $\left.h_{a}\right)$. Take $X, Y \in T_{q} U_{(a)}\left(q \in U_{(a)}\right)$. Since $U_{(a)}$ is totally geodesic in $U, h_{a}(X, Y)=h(X, Y)$ holds. Also, by the assumption, we have

$$
\begin{aligned}
h(X, Y) & =\sum_{b=1}^{r} \varepsilon_{b}^{\frac{1}{b}}\left\langle h(X, Y), E_{b}\right\rangle E_{b} \\
& =\sum_{b=1}^{r} \varepsilon_{b}^{1}\left\langle A_{E_{b}} X, Y\right\rangle E_{b} \\
& =\langle X, Y\rangle \varepsilon_{a}^{\perp} \lambda_{a} E_{a} .
\end{aligned}
$$

Thus we obtain that $h_{a}(X, Y)=\langle X, Y\rangle \varepsilon_{a}^{1} \lambda_{a} E_{a}$, that is, $U_{(a)}$ is a totally umbilical submanifold of $R_{\nu}^{n+r}$ with the mean curvature vector $\varepsilon_{a}^{1} \lambda_{a} E_{a}$. Similarly, we can show that the each maximal integral manifold of $D_{0}$ is a totally geodesic semiRiemannian submanifold of $R_{\nu}^{n+r}$.

Q.E.D.

\section{§4. Proper isoparametric semi-Riemannian submanifolds in a semi-Euclidean space.}

In this section, we characterize proper isoparametric semi-Riemannian submanifolds in a semi-Euclidean space under the hypothesis as in Theorem 2.3. Now we prepare the following lemma.

LEMMA 4.1. Let $M^{n}$ be a semi-Riemannian submanifold of $R_{\nu}^{n+r}$ with the second fundamental form $h$ and $D_{1}, \cdots, D_{t}$ non-degenerate parallel distributions on $M$ such that $T M=D_{1} \oplus \cdots \oplus D_{t}$. Suppose that $h(X, Y)=0$ holds for any $X \in$ $\left(D_{a}\right)_{p}$ and any $Y \in\left(D_{b}\right)_{p}(a \neq b, p \in M)$ and the each maximal integral manifold of $D_{a}(1 \leqq a \leqq t)$ is a totally umbilical submanifold of $R_{\nu}^{n+r}$ with the mean curvature vector $\eta_{a}$. Then

(i) $\tilde{\nabla}_{X} Y \in D_{b}$ for any $X \in D_{a}$ and any $Y \in \Gamma\left(D_{b}\right)(a \neq b)$,

(ii) $\tilde{\nabla}_{X} \eta_{b}=0$ for any $X \in D_{a}(a \neq b)$,

(iii) $\left\langle\eta_{a}, \eta_{b}\right\rangle=0(a \neq b)$.

Proof. It is sufficient to prove the case where $t=2$.

(i) Take $X \in\left(D_{1}\right)_{p}$ and $Y \in \Gamma\left(D_{2}\right)(p \in M)$. Let $\left(U, x_{1}, \cdots, x_{n_{1}}, y_{1}, \cdots, y_{n_{2}}\right)$ be a coordinate neighborhood of $p$ in $M$ such that $\partial / \partial x_{i} \in D_{1}$ and $\partial / \partial y_{0} \in D_{2}$ $\left(1 \leqq i \leqq n_{1}, 1 \leqq j \leqq n_{2}\right)$, where $n_{a}=\operatorname{dim} D_{a}(a=1,2)$. Choose constants $X^{i}\left(1 \leqq i \leqq n_{1}\right)$ 
and smooth functions $Y^{j}\left(1 \leqq j \leqq n_{2}\right)$ such that $X=\sum_{i=1}^{n_{1}} X^{i} \partial / \partial x_{i}$ and $Y=\sum_{j=1}^{n_{2}} Y^{j} \partial / \partial y_{j}$. Since $D_{1}, D_{2}$ are parallel on $M$ and $\nabla_{\partial / \partial x_{i}} \partial / \partial y_{j}=\nabla_{\partial / \partial y_{j}} \partial / \partial x_{i}$, we have $\nabla_{\partial / \partial x_{i}} \partial / \partial y_{j}$ $=0$. Therefore, the assumption on $h$ implies $\tilde{\nabla}_{\partial / \partial x_{i}} \partial / \partial y_{j}=0$ and hence $\tilde{\nabla}_{X} Y$ $=\sum_{i=1}^{n_{1}} \sum_{j=1}^{n_{2}} X^{i}\left(\partial / \partial x_{i} Y^{j}\right) \partial / \partial y_{j} \in\left(D_{2}\right)_{p}$.

(ii) Take $X \in \Gamma\left(D_{1}\right)$. By the Weingarten formula (1.2), we have

$$
\tilde{\nabla}_{X} \eta_{2}=-A_{\eta_{2}} X+\nabla_{X}^{\frac{1}{X}} \eta_{2},
$$

where $A$ and $\nabla^{\perp}$ are the shape operator and the normal connection of $M$, respectively. For $Y \in T_{p} M$, we have

$$
\begin{aligned}
\left\langle A_{\eta_{2}} X, Y\right\rangle & =\left\langle h(X, Y), \eta_{2}\right\rangle \\
& =\left(1 / n_{2}\right) \sum_{j=1}^{n_{2}} \varepsilon_{j}\left\langle h(X, Y), h\left(e_{j}, e_{j}\right)\right\rangle,
\end{aligned}
$$

where $\left(e_{1}, \cdots, e_{n_{2}}\right)$ is a local orthonormal frame field of $D_{2}$ about $p$ and $\varepsilon_{j}=$ $\left\langle e_{j}, e_{j}\right\rangle\left(1 \leqq j \leqq n_{2}\right)$. On the other hand, from the equations (1.3) and (1.4), it follows that

$$
\left\langle h\langle X, Y\rangle, h\left(e_{j}, e_{j}\right)\right\rangle=\left\langle R\left(Y, e_{j}\right) e_{j}, X\right\rangle+\left\langle h\left(X, e_{j}\right), h\left(Y, e_{j}\right)\right\rangle,
$$

where $R$ is the curvature tensor of $M$. Moreover, by the assumption, the right hand side of (4.3, is equal to zero. Therefore, the equation (4.2) implies $A_{\eta_{2}} X=0$. Also, by the assumptions and the equations (1.3) and (1.5), we have

$$
\begin{aligned}
\nabla_{X}^{\perp} \eta_{2}= & \left(1 / n_{2}\right) \sum_{j=1}^{n_{2}} \varepsilon_{j} \nabla_{X}^{\perp}\left(h\left(e_{j}, e_{j}\right)\right) \\
= & \left(1 / n_{2}\right) \sum_{j=1}^{n_{2}} \varepsilon_{j}\left\{\nabla_{e_{j}}^{\perp}\left(h\left(X, e_{j}\right)\right)-h\left(\nabla_{e_{j}} X, e_{j}\right)\right. \\
& \left.\quad-h\left(X, \nabla_{e_{j}} e_{j}\right)+2 h\left(\nabla_{X} e_{j}, e_{j}\right)\right\} \\
= & \left(2 / n_{2}\right) \sum_{j=1}^{n_{2}} \varepsilon_{j} h\left(\nabla_{X} e_{j}, e_{j}\right) .
\end{aligned}
$$

Moreover, since the each maximal integral manifold of $D_{2}$ is totally geodesic in $M$ and totally umbilic in $R_{\nu}^{n+r}, h\left(\nabla_{X} e_{j}, e_{j}\right)=\left\langle\nabla_{X} e_{j}, e_{j}\right\rangle \eta_{2}=0$ holds. Therefore, $\nabla_{X}^{\frac{1}{x}} \eta_{2}=0$ is induced. Finally, we obtain $\tilde{\nabla}_{X} \eta_{2}=0$.

(iii) Let $\left(\bar{e}_{1}, \cdots, \bar{e}_{n_{1}}\right)$ (resp. $\left(e_{1}, \cdots, e_{n_{2}}\right)$ ) be an orthonormal frame of $\left(D_{1}\right)_{p}$ (resp. $\left.\left(D_{2}\right)_{p}\right)(p \in M)$. By the equation (1.4), we have

$$
\begin{aligned}
\left\langle\eta_{1}, \eta_{2}\right\rangle & =\left(1 / n_{1} n_{2}\right) \sum_{i=1}^{n_{1}} \sum_{j=1}^{n_{2}} \bar{\varepsilon}_{i} \varepsilon_{j}\left\langle h\left(\bar{e}_{i}, \bar{e}_{i}\right), h\left(e_{j}, e_{j}\right)\right\rangle \\
& =\left(1 / n_{1} n_{2}\right) \sum_{i=1}^{n_{1}} \sum_{j=1}^{n_{2}} \bar{\varepsilon}_{i} \varepsilon_{j}\left(\left\langle R\left(\hat{e}_{i}, e_{j}\right) e_{j}, \bar{e}_{i}\right\rangle+\left\langle h\left(\bar{e}_{i}, e_{j}\right), h\left(\bar{e}_{i}, e_{j}\right)\right\rangle\right) .
\end{aligned}
$$


Moreover, the right hand side of this equation is equal to zero by the assumptions. Hence, we obtain $\left\langle\eta_{1}, \eta_{2}\right\rangle=0$.

Q.E. D.

For a semi-Riemannian submanifold $M$, we define the first normal space $N_{p}^{1}$ at $p$ as follows:

$$
N_{p}^{1}=\operatorname{Span}\left\{h(X, Y) \mid X, Y \in T_{p} M\right\} .
$$

A subbundle $N$ of $T^{\perp} M$ is said to be normal parallel is $\nabla_{X}^{\perp} E \in N$ for any $X \in T M$ and any $E \in \Gamma(N)$. The following reduction theorem was proved by Magid [6].

THEOREM C. Let $M^{n}$ be a semi-Riemannian submanifold isometrically immersed into $R_{\nu}^{n+r}$ by $f$. If the first normal spaces constitute a normal parallel subbundle, then there exists a complete $(n+s)$-dimensional totally geodesic submanifold $\bar{M}$ of $R_{\nu}^{n+r}$ such that $f(M) \subset \bar{M}$, where $s$ is the dimension of the first normal spaces.

By using this theorem, he obtained the following result [6], where he also treated the case $\langle\eta, \eta\rangle=0$.

THEOREM D. Let $M^{n}$ be a totally umbilical submanifold isometrically immersed into $R_{\nu}^{n+r}$ by $f$. Suppose that the mean curvature vector $\eta$ satisfies $\langle\eta, \eta\rangle$ $\neq 0$. Then

(I) If $\langle\eta, \eta\rangle>0$, then $f(M) \subset S_{\mu}^{n}$

(II) If $\langle\eta, \eta\rangle<0$, then $f(M) \subset H_{\mu}^{n}$, where $\mu$ is the index of $M$.

By using Theorem C, D and Lemma 4.1, we can show the following lemma.

LEMMA 4.2. Under the same hypothesis as in Lemma 4.1, moreover suppose that $\eta_{a}(1 \leqq a \leqq t)$ are non-null and $\left.\left\langle\eta_{a}, \eta_{a}\right\rangle\right\rangle 0(1 \leqq a \leqq u),\left\langle\eta_{a}, \eta_{a}\right\rangle\langle 0(u+1 \leqq a \leqq s)$ and $\eta_{a}=0(s+1 \leqq a \leqq t)$. Then

$$
\begin{gathered}
f(M) \subset S_{\nu_{1}}^{n_{1}}\left(c_{1}\right) \times \cdots \times S_{\nu_{u}}^{n}\left(c_{u}\right) \times H_{\nu_{u+1}}^{n_{u+1}}\left(c_{u+1}\right) \times \cdots \times H_{\nu_{s}}^{n_{s}}\left(c_{s}\right) \times R_{\nu_{0}}^{n_{0}} \\
\subset R_{\nu_{1}}^{n_{1}+1} \times \cdots \times R_{\nu_{u}}^{n_{u}+1} \times R_{\nu_{u+1}}^{n_{u+1}+1+1} \times \cdots \times R_{\nu_{s}+1}^{n_{s}+1} \times R_{\nu_{0}}^{n_{0}} \subset R_{\nu}^{n+r},
\end{gathered}
$$

where $c_{a}=\left\langle\eta_{a}, \eta_{a}\right\rangle,\left(\nu_{a}, n_{a}-\nu_{a}\right)$ is the signature of $D_{a}(1 \leqq a \leqq s)$ and $\left(\nu_{0}, n_{0}-\nu_{0}\right)$ is that of $D_{s+1} \oplus \cdots \oplus D_{t}$.

Proof. We shall prove in the case where $t=3, u=1$ and $s=2$. We denote the maximal integral manifold of $D_{a}$ (resp. $D_{a}^{+}$) through $p \in M$ by $\left(L_{a}\right)_{p}$ (resp. $\left.\left(L_{a}^{\frac{1}{a}}\right)_{p}\right)(1 \leqq a \leqq 3)$, where $D_{a}^{\perp}$ is the orthogonal complement of $D_{a}$ in $T M$. Since 
$\left(L_{1}\right)_{p}$ is a totally umbilical submanifold of $R_{\nu}^{n+r}$ with the mean curvature vector $\eta_{1}$, it is contained in the affine subspace $\left(\bar{L}_{1}\right)_{p}=T_{p}\left(\left(L_{1}\right)_{p}\right) \oplus R\left(\eta_{1}\right)_{p}$ through $f(p)$ by Theorem $\mathrm{C}$, where $R\left(\eta_{1}\right)_{p}$ is the line tangent to $\left(\eta_{1}\right)_{p}$. Now we shall show that $\left(\bar{L}_{1}\right)_{p}$ and $\left(\bar{L}_{1}\right)_{q}$ are parallel in $R_{\nu}^{n+r}$ for any $p, q \in M$. First we consider the case where $p$ and $q$ are contained in a cubic coordinate neighborhood $V$ with respect to $D_{1} \oplus D_{1}^{\frac{1}{1}}$. Then it is clear that $\left(L_{1}^{\frac{1}{1}}\right)_{p} \cap\left(L_{1}\right)_{q} \neq \varnothing$. Take $q^{\prime} \in$ $\left(L_{1}^{\frac{1}{1}}\right)_{p} \cap\left(L_{1}\right)_{q}$. Since $\left(L_{1}^{\frac{1}{1}}\right)_{p}=\left(L_{1}^{\frac{1}{1}}\right)_{q^{\prime}},\left(\bar{L}_{1}\right)_{p}$ and $\left(\bar{L}_{1}\right)_{q^{\prime}}\left(=\left(\bar{L}_{1}\right)_{q}\right)$ are parallel in $R_{\nu}^{n+r}$ by (i), (ii) of Lemma 4.1. Next we consider a general case for $p$ and $q$. Take a curve $\sigma:[0,1] \rightarrow M$ with $\sigma(0)=p, \sigma(1)=q$. Since $\sigma([0,1])$ is compact, there exists a finite open covering $\left\{V_{i} \mid 1 \leqq i \leqq k\right\}$ of $\sigma([0,1])$ by cubic coordinate neighborhoods such that $V_{i} \cap V_{i+1} \neq \varnothing(1 \leqq i \leqq k-1), p \in V_{1}$ and $q \in V_{k}$. Take $p_{i} \in V_{i} \cap V_{i+1}(1 \leqq i \leqq k-1)$. Since $p_{i-1}$ and $p_{i}$ is contained in a cubic coordinate neighborhood, $\left(\bar{L}_{1}\right)_{p_{i-1}}$ and $\left(\bar{L}_{1}\right)_{p_{i}}$ are parallel in $R_{\nu}^{n+r}$. Similarly, so are $\left(\bar{L}_{1}\right)_{p}$ and $\left(\bar{L}_{1}\right)_{p_{1}}$ (resp. $\left(\bar{L}_{1}\right)_{p_{k-1}}$ and $\left.\left(\bar{L}_{1}\right)_{q}\right)$. Therefore, $\left(\bar{L}_{1}\right)_{p}$ and $\left(\bar{L}_{1}\right)_{q}$ are parallel in $R_{\nu}^{n+r}$. Similarly, $\left(\bar{L}_{a}\right)_{p}$ and $\left(\bar{L}_{a}\right)_{q}(a=2,3)$ are parallel in $R_{\nu}^{n+r}$ for any $p, q \in M$, where $\left(\bar{L}_{2}\right)_{p}=T_{p}\left(\left(L_{2}\right)_{p}\right) \oplus R\left(\eta_{2}\right)_{p},\left(\bar{L}_{3}\right)_{p}=T_{p}\left(\left(L_{3}\right)_{p}\right)$. Also, by (iii) of Lemma 4.1, $\left(\bar{L}_{a}\right)_{p} \perp\left(\bar{L}_{b}\right)_{p}$ holds for any $p \in M(a \neq b)$.

Let $R_{(a)}(1 \leqq a \leqq 3)$ be the subspace of $R_{\nu}^{n+r}$ spanned by all tangent vectors of $\left(\bar{L}_{a}\right)_{p}$. Note that $R_{(a)}(1 \leqq a \leqq 3)$ are well-defined and orthogonal to one another by the above facts. Let $R_{(0)}$ be the orthogonal complement of $R_{(1)} \oplus$ $R_{(2)} \oplus R_{(3)}$. We regard $R_{(a)}(0 \leqq a \leqq 3)$ as the affine subspace through the origin of $R_{\nu}^{n+r}$. It is clear that $R_{\nu}^{n+r}=R_{(0)} \times \cdots \times R_{(3)}$. Let $\phi_{a}(0 \leqq a \leqq 3)$ be the natural projections of $R_{\nu}^{n+r}$ onto $R_{(a)}$. It is easy to show that $\phi_{0} \circ f$ is a constant map. Suppose that $\left(L_{\frac{1}{1}}\right)_{p}=\left(L_{1}^{\frac{1}{1}}\right)_{q}$. Then we have $\left(\phi_{1} \circ f\right)(p)=\left(\phi_{1} \circ f\right)(q)$. Since $\left(\eta_{1}\right)_{p}$ and $\left(\eta_{1}\right)_{q}$ are parallel in $R_{\nu}^{n+r}$ by (ii) of Lemma 4.1, $\left(\phi_{1}\right)_{*}\left(\eta_{1}\right)_{p}=\left(\phi_{1}\right)_{*}\left(\eta_{1}\right)_{q}$. Therefore, from Theorem $\mathrm{D}$ and $\left.\left\langle\eta_{1}, \eta_{1}\right\rangle\right\rangle 0$, if follows that there exists a hypersurface $S_{\nu_{1}}^{n_{1}}$ of $R_{(1)}$ which contains both $\left(\phi_{1} \circ f\right)\left(\left(L_{1}\right)_{p}\right)$ and $\left(\phi_{1} \circ f\right)\left(\left(L_{1}\right)_{q}\right)$. By the same method as used in the proof of parallelism between $\left(\bar{L}_{a}\right)_{p}$ and $\left(\bar{L}_{a}\right)_{q}$, we can show that $\left(\phi_{1} \circ f\right)\left(\left(L_{1}\right)_{p}\right)$ is contained in this hypersurface for any $p \in M$. This fact implies that $\left(\psi_{1} \circ f\right)(M) \subset S_{\nu_{1}}^{n_{1}}$. Similar arguements on $\left(\psi_{2} \circ f\right)(M)$ and $\left(\psi_{3} \circ f\right)(M)$ lead to

$$
\begin{array}{r}
f(M) \subset\left(\psi_{1} \circ f\right)(M) \times\left(\psi_{2} \circ f\right)(M) \times\left(\psi_{3} \circ f\right)(M) \subset S_{\nu_{1}}^{n_{1}} \times H_{\nu_{2}}^{n_{2}} \times R_{\nu_{0}}^{n_{0}} \\
\subset R_{(1)} \times R_{(2)} \times R_{(3)} .
\end{array}
$$

Q.E.D.

REMARK. From the assumption of Lemma 4.2, we can show that the second fundamental form is parallel and the normal connection is flat. In [6], 
he characterized a complete Riemannian submanifold $M^{n}$ of $R_{\nu}^{n+r}$ with parallel second fundamental form and flat normal connection. The proof depends on Satz 2 of [12], which uses the Moore's lemma [8]. We can show that they are generally valid for proper semi-Riemannian submanifolds. On the other hand, Moore treats the case where $M$ is a product manifold. If $M$ is complete, then we can use the Moore's lemma for the universal covering of $M$. However, if $M$ is not complete, then the lemma is not valid for this arguement at least globally. The lemma assures that each product neighborhood $V$ of $M$ is contained in a product manifold $\bar{M}$ of semi-Riemannian space forms as an open submanifold. However, we have to show that the manifolds $\bar{M}$ can be tahen in common for all $V$ as in Lemma 4.2.

The distributions $D_{a}(0 \leqq a \leqq s)$ of Theorem 3.1 satisfy the conditions of Lemma 4.2. Hence we have the following proposition.

PROPOSITION 4.3. Let $M^{n}$ be a semi-Riemannian submanifold isometrically immersed into $R_{\nu}^{n+r}$ by $f$. Suppose that there exists a parallel orthonormal normal frame field $\left(E_{1}, \cdots, E_{r}\right)$ on $M$ with the property (\#) in Theorem 2.3. Then

$$
\begin{gathered}
\left.f(M) \subset S_{\nu_{1}}^{n_{1}}\left(c_{1}\right) \times \cdots \times S_{\nu_{u}}^{n_{u}^{u}}\left(c_{u}\right) \times H_{\nu u+1}^{n_{u+1}\left(c_{u+1}\right.}\right) \times \cdots \times H_{\nu_{s}}^{n_{s}}\left(C_{s}\right) \times R_{\nu_{0}}^{n_{0}} \\
\subset R_{\nu_{1}}^{n_{1}+1} \times \cdots \times R_{\nu_{u}^{u}}^{n_{u}+1} \times R_{\nu_{u+1}+1}^{n_{u+1+1}} \times \cdots \times R_{\nu_{s}+1}^{n_{s+1}+1} \times R_{\nu_{0}}^{n_{0}} \subset R_{\nu}^{n+r},
\end{gathered}
$$

where $u$ is the number of +1 in $\left\{\left\langle E_{1}, E_{1}\right\rangle, \cdots,\left\langle E_{s}, E_{s}\right\rangle\right\}$ and $n=n_{0}+\cdots+n_{s}$.

By taking the universal semi-Riemannian covering manifold of $M$ if necessary, this proposition together with Theorem 2.4 gives the following main theorem.

TNEOREM 4.4. Let $M^{n}$ be a proper isoparametric semi-Riemannian submanifold isometrically immersed into $R_{\nu}^{n+r}$ by $f$ with parallel mean curvature vector and $\left\langle\nabla^{\prime} A, \nabla^{\prime} A\right\rangle \geqq 0$. Furthermore, suppose that all sectional curvatures of $M$ are non-negative (resp. non-positive), $\langle\rangle \mid, r_{\Gamma_{M}}$ is positive definite (resp. negative definite). Then

$$
f(M) \subset S_{\nu_{1}}^{n_{1}} \times \cdots \times S_{\nu_{s}}^{n_{s}} \times R_{\nu_{0}}^{n_{0}} \subset R_{\nu_{1}}^{n_{1}+1} \times \cdots \times R_{\nu_{s}}^{n_{s}+1} \times R_{\nu_{0}}^{n_{0}} \subset R_{\nu}^{n+r}
$$

(resp. $\left.f(M) \subset H_{\nu_{1}}^{n_{1}} \times \cdots \times H_{\nu_{g}}^{n_{g}} \times R_{\nu_{0}}^{n_{0}} \subset R_{\nu_{1}+1}^{n_{1}+1} \times \cdots \times R_{\nu_{g}+1}^{n_{g}+1} \times R_{\nu_{0}}^{n_{0}} \subset R_{\nu}^{n+r}\right)$, where $n=$ $n_{0}+\cdots+n_{s}$. 


\section{§5. Proper isoparametric semi-Riemannian submanifolds in $S_{\nu}^{n+r}(c)$ or $H_{\nu}^{n+r}(\tilde{c})$.}

In this section we shall show the results corresponding to $\S 4$ in the case where the ambient space is $H_{\nu}^{n+r}(\tilde{c})\left(\right.$ or $\left.S_{\nu}^{n+r}(\tilde{c})\right)$.

LEMMA 5.1. Let $M^{n}$ be a proper isoparametric semi-Riemannian submanifold of $H_{\nu}^{n+r}(\tilde{c})$ such that

(i) the mean curvature vector is parallel,

(ii) $\left\langle\nabla^{\prime} A, \nabla^{\prime} A\right\rangle \geqq 0$.

Then, if we consider $M$ as isometrically immersed into $R_{\nu+1}^{n+r+1}, M$ also is a proper isoparametric semi-Riemannian submanifold with (i) and (ii).

Proof. Let $A$ and $\nabla^{\perp}$ (resp. $\tilde{A}$ and $\tilde{\nabla}^{\perp}$ ) be the shape operator and the normal connection of $M$ in $H_{\nu}^{n+r}(\tilde{c})$ (resp. $R_{\nu+1}^{n+r+1}$ ). By the Gauss formula (1.1) and the Weingaten formula (1.2), we have

$$
\begin{aligned}
& \tilde{A}_{E} X=A_{E} X, \quad \tilde{\nabla}_{X}^{1} E=\nabla_{\bar{X}}^{\perp} E, \\
& \tilde{A}_{\bar{E}} X= \pm \sqrt{-\tilde{c}} X, \quad \tilde{\nabla}_{X}^{\perp} \bar{E}=0
\end{aligned}
$$

for any $X \in T M$ and any $E \in \Gamma\left(T^{\perp} M\right)$, where $\bar{E}$ is a unit normal vector field of $H_{\nu}^{n+r}(\tilde{c})$ in $R_{\nu+1}^{n+r+1}$ and $T^{\perp} M$ is the normal bundle of $M$ in $H_{\nu}^{n+r}(\tilde{c})$. By (5.1), (5.2) and the assumption, we see that $M$ is a proper isoparametric semi-Riemannian submanifold of $R_{\nu+1}^{n+r+1}$.

Let $\eta$ (resp. $\tilde{\eta}$ ) be the mean curvature vector of $M$. in $H_{\nu}^{n+r}(\tilde{c})$ (resp. $R_{\nu+1}^{n+r+1}$ ) and $\bar{\eta}$ that of $H_{\nu}^{n+r}(\tilde{c})$ in $R_{\nu+1}^{n+r+1}$. Since $H_{\nu}^{n+r}(\tilde{c})$ is a totally umbilical submanifold of $R_{\nu+1}^{n+r+1}, \tilde{\eta}=\eta+\bar{\eta}$ holds. Moreover, the equation (5.1) and the assumption (resp. the equation (5.2) and $\bar{\eta}= \pm \sqrt{-\tilde{c}} \bar{E}$ ) imply $\tilde{\nabla}_{\frac{1}{X}}^{\frac{1}{\eta}}=0$ (resp. $\tilde{\nabla}_{\bar{X}}^{\frac{1}{\eta}} \bar{\eta}$ $=0)$ for any $X \in T M$. Thus $\tilde{\nabla}_{\frac{1}{X}}^{1} \tilde{\eta}=0$.

By (5.1), (5.2) and the assumption, we can show $\left\langle\tilde{\nabla}^{\prime} \tilde{A}, \tilde{\nabla}^{\prime} \tilde{A}\right\rangle=\left\langle\nabla^{\prime} A, \nabla^{\prime} A\right\rangle$ $\geqq 0$, where $\left(\tilde{\nabla}_{X}^{\prime} \tilde{A}\right)_{E} Y=\nabla_{X}\left(\tilde{A}_{E} Y\right)-\tilde{A}_{\tilde{\nabla}_{X} E_{E}} Y-\tilde{A}_{E}\left(\nabla_{X} Y\right)$ for any $X \in T M$, any $Y \in$ $\Gamma(T M)$ and any $E \in \Gamma\left(T^{\perp} M \oplus T^{\perp} H_{\nu}^{n+r}(\tilde{c})\right)$.

Q.E.D.

This lemma together with Theorem 4.4 gives the following theorem.

THEOREM 5.2. Let $M^{n}$ be a proper isoparametric semi-Riemannian submanifold isometrically immersed into $H_{\nu}^{n+r}(\tilde{c})$ by $f$ with parallel mean curvature vector and $\left\langle\nabla^{\prime} A, \nabla^{\prime} A\right\rangle \geqq 0$. Furthermore, suppose that all sectional curvatures of $M$ are non-positive, $\langle\rangle \mid,{ }_{T^{\perp} M}$ is negative definite. Then 


$$
(i \circ f)(M) \subset H_{\nu 1}^{n_{1}}\left(c_{1}\right) \times \cdots \times H_{\nu s}^{n_{s}}\left(c_{s}\right) \subset H_{\nu+s-r-1}^{n+s-1}(\bar{c}) \subset H_{\nu}^{n+r}(\tilde{c}) \subset R_{\nu+1}^{n+r+1},
$$

where $n=n_{1}+\cdots+n_{s}, 1 / c_{1}+\cdots+1 / c_{s}=1 / \bar{c} \geqq 1 / \tilde{c}$ and $i$ is the inclusion mapping of $H_{\nu}^{n+r}(\tilde{c})$ into $R_{\nu+1}^{n+r+1}$.

Proof. By Theorem 4.4 and Lemma 5, 1, we have

$$
\begin{aligned}
& (i \circ f)(M) \subset H_{\nu_{1}}^{n_{1}}\left(c_{1}\right) \times \cdots \times H_{\nu_{s}}^{n_{s}}\left(c_{s}\right) \times R_{\nu_{0}}^{n_{0}} \times\{x\} \\
& \quad \subset R_{\nu_{1}+1}^{n_{1}+1} \times \cdots \times R_{\nu_{s}+1}^{n_{s}+\mathfrak{I}} \times R_{\nu_{0}}^{n_{0}} \times R_{r-s+1}^{r-s+1}=R_{\nu+1}^{n+r+1} .
\end{aligned}
$$

Take $p \in(i \circ f)(M)$. We denote the leaf of $R_{\nu_{0}}^{n_{0}}$ through $p$ by $L_{p}$ and $L_{p} \cap$ $(i \circ f)(M)$ by $\hat{L}_{p}$. Suppose $n_{0}>1$. Since $\hat{L}_{p}$ is totally geodesic in $R_{\nu+1}^{n+r+1}$, it is also totally geodəsic in $H_{\nu}^{n+r}(\tilde{c})$. Hence $\hat{L}_{p}$ is of constant curvature $\tilde{c}$. This fact contradicts the flatness of $L_{p}$. Therefore, we have $n_{0} \leqq 1$. If $n_{0}=1$, then $\hat{L}_{p}$ is a family of non-null curves of $H_{\nu}^{n+r}(\tilde{c})$. By the way, all line segments of $R_{\nu+1}^{n+r+1}$ contained in $H_{\nu}^{n+r}(\tilde{c})$ are null. Hence each component of $\hat{L}_{p}$ is not a line segment. This fact contradicts that $L_{p}$ is totally geodesic in $R_{\nu+1}^{n+r+1}$. Thus we see that $n_{0}=0$.

Let $o_{a}$ be the center of $H_{\nu_{a}^{n}}^{n}\left(c_{a}\right)(1 \leqq a \leqq s)$. Take $p \in(i \circ f)(M)$. We can uniquely decompose $p$ into $p=p_{1}+\cdots+p_{s}+x$, where $p_{a} \in R_{\nu a+1}^{n a+1}(1 \leqq a \leqq s)$. From $\left\langle p_{a}-o_{a}, p_{a}-o_{a}\right\rangle=1 / c_{a}$, it follows that

$$
\begin{aligned}
\left\langle p_{a}, p_{a}\right\rangle & =\left\langle o_{a}+\left(p_{a}-o_{a}\right), o_{a}+\left(p_{a}-o_{a}\right)\right\rangle \\
& =\left\langle o_{a}, 2 p_{a}-o_{a}\right\rangle+1 / c_{a} \\
& =\left\langle o_{a}, 2 p-o\right\rangle+1 / c_{a},
\end{aligned}
$$

where $o=o_{1}+\cdots+o_{s}$. Hence we have

$$
\begin{aligned}
1 / \tilde{c}=\langle p, p\rangle & =\left\langle p_{1}, p_{1}\right\rangle+\cdots+\left\langle p_{s}, p_{s}\right\rangle+\langle x, x\rangle \\
& =\langle 0,2 p-0\rangle+1 / c_{1}+\cdots+1 / c_{s}+\langle x, x\rangle .
\end{aligned}
$$

Thus $\langle 0,2 p-0\rangle=1 / \tilde{c}-\left(1 / c_{1}+\cdots+1 / c_{s}+\langle x, x\rangle\right)$ holds. This equality implies that $\langle p, o\rangle$ is independent of $p \in(i \circ f)(M)$. Hence, if $o$ is a non-zero vector, then $(i \circ f)(M)$ is contained in the hyperplane orthogonal to 0 in $R_{\nu_{1}+1}^{n_{1}+1} \times \cdots$ $\times R_{\nu_{s}+1}^{n_{s}+1} \times\{x\}$. This fact contradicts that $(i \circ f)(M)$ is full in $R_{\nu_{1}+1}^{n_{1}+1} \times \cdots \times R_{\nu_{s}+1}^{n_{s}+1}$ $\times\{x\}$. Therefore, we see that $o$ is the zero vector and $1 / \tilde{c}=1 / c_{1}+\cdots+1 / c_{s}$ $+\langle x, x\rangle$. These facts imply that

and hence

$$
H_{\nu_{1}}^{n_{1}}\left(c_{1}\right) \times \cdots \times H_{\nu_{s}}^{n_{s}}\left(c_{s}\right) \times\{x\} \subset H_{\nu}^{n+r}(\tilde{c})
$$




$$
\begin{aligned}
H_{\nu_{1}}^{n_{1}}\left(c_{1}\right) \times \cdots \times H_{\nu_{s}}^{n_{s}}\left(c_{s}\right) \times\{x\} & \subset H_{\nu}^{n+r}(\tilde{c}) \cap\left(R_{\nu_{1}+1}^{n_{1}+1} \times \cdots \times R_{\nu_{s}+1}^{n_{s}+1} \times\{x\}\right) \\
& =H_{\nu+s-r-1}^{n+s-1}(\bar{c}) \times\{x\} .
\end{aligned}
$$

Here $1 / \bar{c}=1 / c_{1}+\cdots+1 / c_{s}$ because

$$
1 / \tilde{c}=\langle q, q\rangle=\langle x+(q-x), x+(q-x)\rangle=\langle x, x\rangle+1 / \bar{c}
$$

for $q \in H_{\nu+s-r-1}^{n+s-1}(\bar{c}) \times\{x\}$. Therefore, we obtain

$$
\begin{array}{r}
(i \circ f)(M) \subset H_{\nu_{1}}^{n_{1}}\left(c_{1}\right) \times \cdots \times H_{\nu_{s}}^{n_{s}}\left(c_{s}\right) \times\{x\} \subset H_{\nu+s-r-1}^{n+s-1}(\bar{c}) \times\{x\} \\
\subset H_{\nu}^{n+r}(\tilde{c}) \subset R_{\nu+1}^{n+r+1} .
\end{array}
$$

Q.E.D.

Similarly, in the case where the ambient space is $S_{\nu}^{n+r}(\tilde{c})$, we have the following theorem.

THEOREM 5.3. Let $M^{n}$ be a proper isoparametric semi-Riemannian submanifold isometrically immersed into $S_{\nu}^{n+r}(\tilde{c})$ by $f$ with parallel mean curvature vector and $\left\langle\nabla^{\prime} A, \nabla^{\prime} A\right\rangle \geqq 0$. Furthermore, suppose that all sectional curvatures of $M$ are non-negative, $\left.\langle\rangle\right|_{,T^{\perp} M}$ is positive definite. Then

$$
(i \circ f)(M) \subset S_{\nu_{1}}^{n_{1}}\left(c_{1}\right) \times \cdots \times S_{\nu_{s}}^{n_{s}}\left(c_{s}\right) \subset S_{\nu}^{n+s-1}(\bar{c}) \subset S_{\nu}^{n+r}(\tilde{c}) \subset R_{\nu}^{n+r+1},
$$

where $n=n_{1}+\cdots+n_{s}, 1 / c_{1}+\cdots+1 / c_{s}=1 / \bar{c} \leqq 1 / \tilde{c}$ and $i$ is the inclusion mapping of $S_{\nu}^{n+r}(\tilde{c})$ into $R_{\nu}^{n+r+1}$.

\section{References}

[1] Abe, N., Koike, N. and Yamaguchi, S., Congruence theorems for proper semiRiemannian hypersurfaces in a real space form, Yokohama Math. J. 35 (1987), 123-136.

[2] Erbacher, J., Isometric immersions of constant mean curvature and triviality of the normal connection, Nagoya Math. J. 45 (1971), 139-165.

[3] Hahn, J., Isoparametric hypersurfaces in the pseundo-Riemannian space forms, Math. Z. 187 (1984), 195-208.

[4] Kobayashi, S. and Nomizu, K., Foundations of Differential Geometry, Volume I, Interscience Tracts No. 15, John Wiley and Sons, New York, 1963.

[5] Lawson, Jr. H. B., Local rigidity theorems for minimal hypersurfaces, Ann. of Math. 89 (1969), 187-197.

[6] Magid, M.A., Isometric immersions of Lorentz space with parallel second fundamental forms, Tsukuba J. Math. 8 (1984), 31-54.

[7] Magid, M. A., Lorentzian isoparametric hypersurfaces, Pacific J. Math. 118 (1985), 165-197.

[8] Moore, J. D., Isometric immersions of Riemannian products, J. Differential Geom. 5 (1971), 159-168.

[9] O'Neill, B., Semi-Riemannian Geometry, with Applications to Relativity, Academic Press, New York, 1983. 
[10] Petrov, A.Z., Einstein Spaces, Pergamon Press, Hungary, 1969.

[11] Terng, C.L., Isoparametric submanifolds and their coxeter groups, J. Differential Geom. 21 (1985), 79-107.

[12] Walden, R., Untermannigfaltigkeiten mit parallel zweiter Fnudamentalform in euklidischen Raumen and Sphären, Manuscripta Math. 10 (1973), 91-102.

[13] Wu, H., On the de Rham decomposition theorem, Illinois J. Math. 8 (1964), 291311.

[14] Yano, K. and Ishihara, S., Submanifolds with parallel mean curvature vector, J. Differential Geom. 6 (1971), 95-118.

[15] Yano, K. and Kon, M., Structures on Manifolds, World Scientific Publishing Co. Pte. Ltd., Singapore, 1984.

\author{
Department of Mathematics \\ Faculty of Science \\ Science University of Tokyo \\ Tokyo 162 Japan
}

